\title{
INTERFERENCE FRINGES IN SYNCHROTRON SECTION TOPOGRAPHY OF IMPLANTED SILICON WITH A VERY LARGE ION RANGE
}

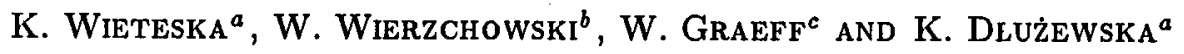 \\ ${ }^{a}$ Institute of Atomic Energy, 05-400 Otwock-Świerk, Poland \\ ${ }^{b}$ Institute of Electronic Materials Technology, Wólczyńska 133, 01-919 Warsaw, Poland \\ ${ }^{c}$ HASYLAB at DESY, Notkestr. 85, 22603 Hamburg, Germany
}

\begin{abstract}
Silicon crystals implanted with $9 \mathrm{MeV}$ protons to the dose of $5 \times 10^{17} \mathrm{~cm}^{-2}$ were studied with $\mathrm{X}$-ray topographic methods using both conventional and synchrotron radiation sources. After the implantation the crystals were thermally and electron annealed. The implantation produced large $600 \mu \mathrm{m}$ thick shot-through layer while the total thickness of the samples was $1.6 \mathrm{~mm}$. It was confirmed by means of double crystal topography that the whole crystal was elastically bent. The transmission section patterns revealed both parts of the implanted crystal separated by strong contrasts coming from the most damaged layer and distinct interference fringes which appeared on one side of the topograph only. The location of the fringes changed when the beam entered the other side of the sample. The mechanism of fringe formation was studied with numerical integration of the Takagi-Taupin equations, especially studying the intensity distribution in the diffraction plane. The simulations reproduced the location of the fringes in different geometries and indicate that they can be caused both by variable crystal curvature and variable ion dose.
\end{abstract}

PACS numbers: $61.10 .-\mathrm{i}, 61.80 .-\mathrm{x}$

\section{Introduction}

Implanted crystals may produce interference fringes in transmission diffraction geometry. In most cases the fringes are due to diffraction of $\mathrm{X}$-rays by the bicrystal-like nature of the sample consisting of a relatively thin shot-through layer and the bulk, separated by the most damaged region. This type of model was developed by Bonse and Hart [1] and also in [2, 3]. Another mechanism of fringe formation was proposed by Simon and Authier [4] where the diffraction from two regions with different diffraction vectors causes formation of moire type fringes.

The use of the bicrystal models was unsuccessful in the analysis of the fringes observed in the Bragg case [5]. A number of diffraction effects was reasonably reproduced in our former papers [6-8] using numerical integration of the Takagi-Taupin 
equations. In these calculations we assumed the depth distribution of the lattice parameter change to be proportional to the vacancies-interstitials profile obtained from the Biersack-Ziegler theory [9].

In the present paper we discuss a new type of fringes appearing in transmission topographs of relatively thick $(1.6 \mathrm{~mm})$ crystals where the high energy protons produced a very thick $600 \mu \mathrm{m}$ shot-through layer. Some features of the observed fringes could suggest their analogy to moire fringes but the analysis of their formation also required the use of the Takagi-Taupin theory.

\section{Experimental}

The (111) oriented $1.6 \mathrm{~mm}$ thick silicon samples were implanted with $9 \mathrm{MeV}$ protons to the dose of $5 \times 10^{17} \mathrm{~cm}^{-2}$. After the implantation process a mixed thermal processing was realised with two periods of 1 and 3 hours thermal annealing up to $400^{\circ} \mathrm{C}$ and several electron beam irradiations with $1.5 \mathrm{MeV}$ electrons up to the dose of $1.8 \times 10^{18} \mathrm{~cm}^{-2}$.

The double-crystal investigations were performed in parallel arrangement using the 333 and 111 reflections of $\mathrm{Cu} K_{\alpha}$ radiation. The characteristic "zebra" patterns due to the bending of the samples were used to determine the local radius of curvature.

The synchrotron topographic investigations were performed both with section and projection methods in transmission geometry using white beam from the bending magnet of the DORIS ring operating at $4.445 \mathrm{GeV}$. In the section experiments the beam front was limited to $5 \mu \mathrm{m}$. Each exposure provided a number of spots which were indexed and analysed. The topographs were taken with the beam entering the sample either from the substrate or from the surface layer.

\section{Results and discussion}

The double-crystal topographs showed that the whole samples were bent with rather regular radial symmetry and with the curvature systematically decreasing towards the edge of the crystal. The radius of curvature in the center of the implanted area was close to $40 \mathrm{~m}$ and at least twice greater on the boundaries.

The synchrotron section patterns revealed strong direct contrast from the most damaged region; its location corresponded well to the theoretical evaluation of the penetration depth of about $600 \mu \mathrm{m}$. The topographs exhibited distinct interference fringes which are shown in Fig. 1. In some cases the fringes were oriented close to the plane of diffraction but some curvature was always observed.

A characteristic feature of the observed fringes is that they appear only on one side of the topographs. They are visible in the part of the section pattern corresponding to the substrate. When the sample is turned through $180^{\circ}$ and the beam enters from the other side, the fringe pattern changes its position with respect to the incident beam.

Despite some similarities to a moire pattern, the presently discussed fringes were not explainable by a simple model. Some conclusions concerning the formation of the fringes were found using numerical integration of the Takagi-Taupin equations. The most valuable results were provided by the simulation of the intensity distribution in the plane of diffraction. 
(a)

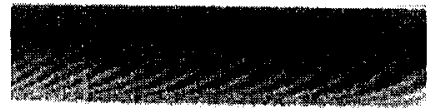

(b)

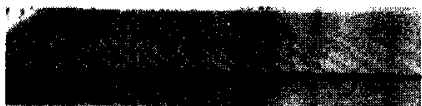

Fig. 1. Synchrotron transmission section topographs of $9 \mathrm{MeV}$ proton implanted silicon in $3 \overline{1} \overline{1}$ reflection selecting $0.7 \AA$ wavelength: (a) X-ray beam entering non-implanted side of the sample; (b) X-ray beam entering implanted part of the crystal.

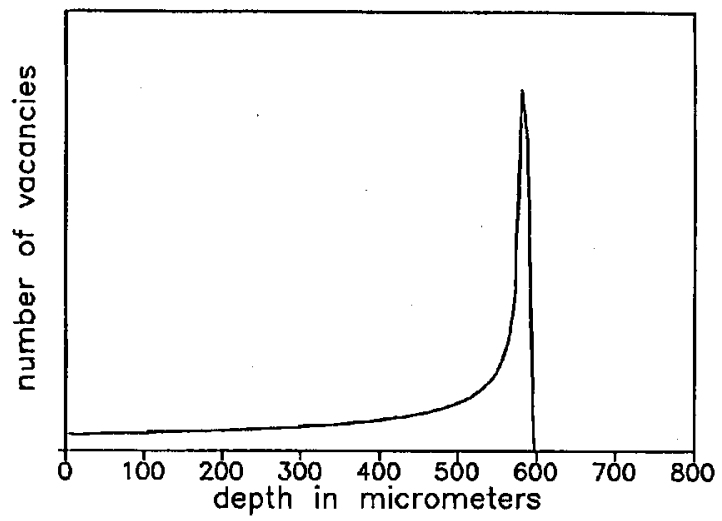

Fig. 2. Lattice parameter depth distribution profile used for numerical simulations of the fringe pattern.

The simulations were performed assuming the depth distribution of the lattice parameter to be proportional to the vacancy distribution calculated using the Monte Carlo method from the Biersack-Ziegler theory [9], shown in Fig. 2. The calculation takes into account both the lattice parameter changes and the misorientation caused by the curvature of the crystal.

The intensity distributions of the reflected wave illustrating the fringe formation are shown in Fig. 3. Figure 3a corresponds to the situation when the beam enters the substrate while Figs. $3 \mathrm{~b}, \mathrm{c}$ correspond to the beam entering the implanted side of the sample. In all pictures the formation of the Pendellösung fringes is visible in the first part of the crystal, close to the entrance of the beam. The crystal curvature and lattice parameter change due to the implantation cause a change in the periodicity of the Pendellösung fringes with respect to the ideal crystal. The system of Pendellösung fringes is destroyed in the most deformed region, which gives also distinct direct contrast.

The simulations confirm the formation of relatively strong interference fringes in the topographs appearing only on one side of the direct contrast, as observed in the experiment. The calculations show that the appearance of the fringes in the experimental topographs can be explained as due to variable curvature. This 

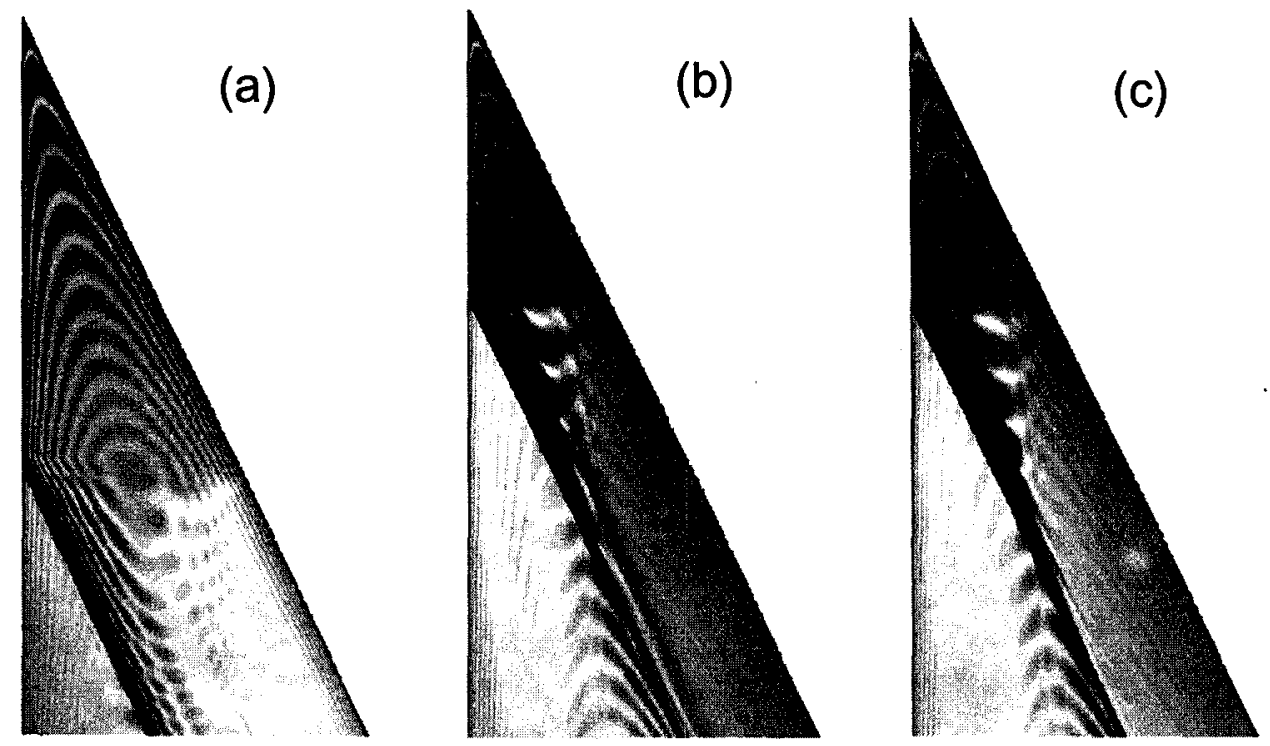

Fig. 3. Numerical simulation of reflected wave intensity aistribution in diffraction plane illustrating formation of the fringes for $3 \overline{1} \overline{1}$ reflection of $0.7 \AA$ wavelength: (a) the beam entering concave non-implanted side of the sample, (b) the beam entering convex implanted side of the sample with the radius of curvature the same as in (a), (c) the beam entering convex implanted side of the sample with the radius of curvature $20 \%$ greater than in (b).

may be followed in Figs. $3 b, c$ which differs by $20 \%$ in the radius of curvature. The periodicity of the fringes can also be affected by the ion dose change, similarly as in [5-7].

The correspondence of the simulation to the experimental topograph is not perfect. The most probable reason for the discrepancy is the possible change in the lattice parameter distribution caused by the annealing process.

\section{References}

[1] U. Bonse, M. Hart, Phys. Status Solidi 33, 351 (1969).

[2] A.O. Aboyan, P.A. Bezirganyan, S.E. Bezirganyan, A.M. Grigoryan, A.S. Tumasyan, Cryst. Res. Technol. 25, 1405 (1990).

[3] A.G. Sedrakyan, V.S. Haroutyunyan, P.H. Bezirganyan, M. Subotowicz, K. Trouni, Phys. Status Solidi A 123, 83 (1991).

[4] D. Simon, A. Authier, Acta Crystallogr. A 24, 527 (1968).

[5] K. Wieteska, W. Wierzchowski, Phys. Status Solidi A 147, 55. (1995).

[6] K. Wieteska, W. Wierzchowski, J. Phys. D 28, A74 (1995).

[7] K. Wieteska, W. Wierzchowski, W. Graeff, submitted to J. Appl. Cryst.

[8] K. Wieteska, W. Wierzchowski, W. Graeff, submitted to Il Nuovo Cimento.

[9] J.F. Ziegler, J.P. Biersack, U. Littmark, in: The Stopping and Range of Ions in Solids, Ed. J.F. Ziegler, Pergamon Press, New York 1985, p. 202. 